\title{
Ambiguous musical figures and auditory streaming
}

\author{
JACQUI SMITH, STEVEN HAUSFELD, RODERICK P. POWER, \\ and ANGELA GORTA \\ Macquarie University, North Ryde, New South Wales, Australia
}

\begin{abstract}
Three experiments with musicians and nonmusicians $(\mathrm{N}=338)$ explored variations of Deutsch's musical scale illusion. Conditions under which the illusion occurs were elucidated and data obtained which supported Bregman's suggestion that auditory streaming results from a competition among alternative perceptual organizations. In Experiment 1, a series of studies showed that it is more difficult to induce the scale illusion than might be expected if it is accepted that an illusion will be present for most observers despite minor changes in stimuli and experimental conditions. The stimulus sequence seems better described as an ambiguous figure. Having discovered conditions under which the scale illusion could be reliably induced, Experiments 2 and 3 manipulated additional properties of the stimulus (timbre, loudness, and tune) to provide cues to streaming other than pitch and location. The data showed that streaming of this sequence can be altered by these properties, supporting the notion of a general parsing mechanism which follows general gestalt principles and allows streaming by many stimulus dimensions. Finally, suggestions are made as to how this mechanism might operate.
\end{abstract}

Listening to music provides an excellent context for exploring the phenomenon of auditory streaming. Bregman and Campbell (1971) described auditory streaming as the perceptual splitting of concurrent auditory events into separate streams or sequences. This must happen, for example, when listening to an orchestra. The listener needs to decide which melodies are being played by what instruments, where the sounds originated, and how many instruments and melodies there are.

Research on auditory streaming has focused on much simpler situations than listening to an orchestra. Bregman and Campbell, for instance, binaurally presented subjects with a rapid repetitive sequence of six different $100-\mathrm{msec}$ pure tones, three high pitched and three low pitched, one and a half octaves apart. Although successive tones alternated from the high- to low-pitch range, subjects organized the high- and low-pitched sounds into separate auditory streams. Some subjects $(59 \%)$ claimed that these two streams were successive (i.e., three high tones followed by three low tones, or vice versa); the remainder reported that the streams were concurrent. Neither of these percepts are veridical.

Deutsch (1975b) found similar effects when she pre-

This study was supported by a Macquarie University research grant to the first author and by assistance from the $S \& \mathrm{~J} R e$ search Foundation. The authors are grateful to Karen Rickard for her assistance in data collection, to the staff and senior students of the N.S.W. Conservatorium of Music High School for their cooperation, to the Conservatorium for permitting us to use their Fairlight Computer Musical Instrument, and to June Crawford and George Cooney for their statistical advice. Thanks are also due to two anonymous reviewers for their helpful comments on an earlier draft. The authors' mailing address is: School of Behavioural Sciences, Macquarie University, North Ryde, N.S.W. 2113, Australia. sented dichotic tonal sequences. Her "musical illusions" occurred because subjects streamed not just by ear of presentation, as might have been predicted from previous work with linguistic material (e.g., Moray, 1969), but also by pitch. One example is her octave illusion. Deutsch (1974) presented subjects with a continuous sequence in which a high tone alternated with a tone an octave lower. This sequence was played simultaneously to each ear except that as one ear received the high tone, the other received the lower tone. None of her 53 right-handed subjects streamed by ear alone, which would have led to veridical perception. Instead, these subjects' percepts reflected a combination of streaming by pitch and by ear: 58\% reported hearing a single tone switching from ear to ear and simultaneously changing pitch, 25\% reported a single-pitch tone oscillating from ear to ear, and $17 \%$ reported various different-pitch tones either oscillating simultaneously or sounding just in one ear.

In another study, Deutsch (1975a) presented subjects with dichotic repetitions of $\mathrm{C}$ major scales in which successive notes of ascending and descending versions of the scale alternated between ears. Figure 1 shows how

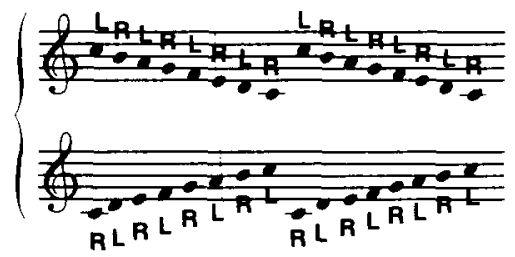

Figure 1. Arrangement of stimulus presentation for Deutsch's scale illusion based on the $C$ major scale. $(L=$ presented to left ear $\mathbf{R}=$ presented to right ear.) 
these ascending and descending $\mathrm{C}$ major scales switched from ear to ear. This arrangement meant that lines 5 and 6 from Figure 2 were presented, one to each ear. (Throughout, specific line numbers mentioned will refer to the lines of Figure 2.) Deutsch's stimulus consisted of pure tones changing pitch every $250 \mathrm{msec}$ with no gaps between the tones. Subjects listened through headphones to 10 continuous repetitions of the sequence. They were instructed to attend to each ear individually "so as to discover which earphone was emitting the different tones" (p. 1156). Subjects gave verbal reports of their percepts and were also asked to shadow-sing each tune they heard.

Deutsch (1975a) reported that $70 \%$ of her subjects heard the high notes ascending and descending in contrary motion to the low notes, that is, lines 3 and 4 in Figure 2. Moreover, for right-handers, the higher notes, line 3 , tended to be localized in the right ear, and the lower notes, line 4 , in the left ear, regardless of headphone position. The remaining $30 \%$ of Deutsch's subjects heard only a part of the effect: for example, $17 \%$ of the right-handers reported hearing only one stream, line 3 . Left-handers were especially varied in their percepts. Only $51 \%$ of left-handers reported hearing both line 3 and line 4 , and these subjects did not all localize the high notes in the right ear. In addition, some left-handers who heard only one stream reported, veridically, that the notes switched from ear to ear.

Deutsch called this phenomenon (i.e., the perception of lines 3 and 4 when lines 5 and 6 had been presented) the "scale illusion." Her explanation for it was that subjects formed auditory streams on the basis of pitch,
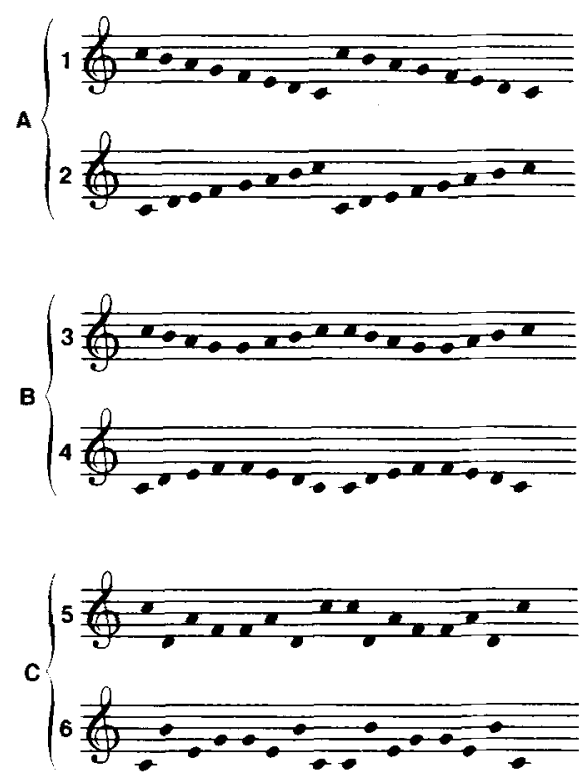

Figure 2. The six (lines 1-6) and three (A, B, and C) response alternatives available to subjects in various phases of Experiments 1,2 , and 3 . and she concluded that, for this tonal sequence, pitch was the most salient feature.

In discussing the processes involved in such examples of auditory streaming, Deutsch $(1979,1980$; Deutsch \& Roll, 1976) proposed that the stimulus attributes, location ("where?") and pitch ("what?"), are analyzed by different mechanisms, with the output from these analyses being integrated at a later stage. Each of these mechanisms is sensitive to the conjunction of frequency and spatial location information, so that the "decision as to what is heard is determined by where the signals are coming from" and the "decision as to where the stimulus is located is determined by what the signal frequencies are" (1980, p. 578). Deutsch emphasizes the role of unconscious inference in the integration of these decisions, taking into account the fact that "in everyday life similar sounds are likely to emanate from the same source and different sounds from different sources" (Deutsch, 1975b, p. 98).

Bregman $(1978 \mathrm{a}, 1978 \mathrm{~b})$ has a similar theory. He explained streaming as part of the listener's preattentive processes (Bregman \& Campbell, 1971) and argued that at this preattentive stage complex acoustic information is decomposed into several auditory streams by a general "parsing mechanism" before more detailed pattern recognition. This parsing mechanism uses gestalt principles to determine the organization of a minimal number of streams that best describe the auditory information (Bregman, 1978b, 1981). The entire process is characterized as a competition among alternative potential organizations. This competition is decided by a complex interaction between the attributes of the stimuli, the listener's general auditory heuristics, and to a lesser extent the listener's particular knowledge, skills, and intentions. Bregman (1978b, p. 64) noted that "no one heuristic will necessarily always succeed, but if there are many of them, competing or reinforcing one another, the right description of the input should generally emerge."

Bregman's account makes it clear that one should expect a wide range of changes in stimulus parameters and listener characteristics to lead to systematic and predictable changes in perception. To date, most auditory streaming research has juxtaposed only two dimensions, pitch and location. It is important to introduce cues to other streaming possibilities (e.g., timbre or loudness), and to include nonphysical dimensions (e.g., the "tune" dimension discussed below), to determine whether streaming can be based on a variety of stimulus dimensions. It is also important to determine if the parsing mechanism reflects a listener's special experience, such as musical training. Both Bregman (1978b) and Deutsch (1975b) have commented that musical experience may play a role in auditory streaming, but neither seems to have tested this. Indeed, there has been little consideration of possible interactions between the characteristics of the listener and those of the stimuli, 
even though there are many reports that, on other musical tasks, musicians and nonmusicians differ (e.g., Deutsch, 1978; Dowling, 1978; Gates \& Bradshaw, 1977; Sloboda, 1976, 1978; Smith, Note 1).

Following a suggestion by Power, Hausfeld, and Gorta (1981), Deutsch's (1975a) scale illusion formed the basis of our investigation into auditory streaming. The tone sequence for this illusion seemed well suited to the manipulation of additional stimulus and subject parameters. Although Deutsch reported that pitch was the dominant streaming cue producing the scale illusion, there are obviously at least two other possible organizations of this sequence, as Figure 2 indicates. If one were to stream by ear (localization cue), then one would hear lines 5 and 6 , while if one were to stream by a well-known scale (tune cue), then one would hear lines 1 and 2. We have used the term "tune" here for ease of reference and to reflect the experiential basis of this cue which is fundamental to western music.

Experiment 1 reports our attempts to reproduce Deutsch's results with various changes in stimulus parameters and procedures, draws attention to the elusive nature of the "illusion," and leads us to propose, instead, that the sequence is best described as an ambiguous figure. Experiment 2 investigates the circumstances under which listeners will cease to stream this sequence by pitch. Judd (1979), using rather different stimuli, had previously demonstrated some conditions in which pitch streaming breaks down. In the present study, variations were introduced to the basic scale illusion sequence with the intention of producing perceived organizations based on the dimensions of spatial location (ear), pitch, loudness, timbre, and "tune." Experiment 3 replicates some aspects of Experiment 2, using binaural presentations. Throughout, the percepts of musicians and nonmusicians are compared.

As a first step in using Deutsch's scale illusion to investigate auditory streaming, we wanted to see whether Deutsch's illusion could be replicated with more usual musical stimuli (i.e., complex rather than pure tones), in different listening conditions, and with listeners varying in musical experience. Deutsch did not indicate the musical experience of her subjects, although her technique of having them shadow-sing the stimuli would be a fairly difficult task for nonmusicians.

It is important to test the robustness of illusory phenomena, and hence their explanations, by varying stimulus parameters and experimental procedures (Coren \& Girgus, 1978). Deutsch and Roll (1976) have reported, for example, that the essential character of the octave illusion remained constant despite changes in the sequential patterning of the sounds, type of sounds, stimulus intensity, and the frequency ratio between the alternating sounds. A previous test of the scale illusion has indicated that this phenomenon will also remain intact despite variations in stimuli and procedure. Butler (1979) reproduced the illusion with notes played on a piano and presented through stereo speakers, rather than with pure tones presented via headphones in the strict dichotic listening procedure. He asked his subjects, who were all musicians, to both write their perception of the sequence in musical notation and to select the best representation from three forced-choice alternatives (A, B, and $\mathrm{C}$ in Figure 2). These changes provide the background for the manipulations adopted in the present study.

Butler's forced-choice procedure seemed a more appropriate measure than the shadow-singing response of Deutsch, especially when testing subjects with varying amounts of musical training. It did, however, seem rather restrictive to limit subjects' alternatives to three, as Butler had. This might well bias the results by raising the "chance" level of illusory responses and by forcing subjects, such as those of Deutsch (1975a) who heard only the high pitched stream (line 3 ), to indicate response $B$ even though they could not hear line 4 . Thus, it seemed preferable to allow subjects all six response alternatives. In addition, subjects were given two blank spaces in which they could draw, or musically notate, some other representation if lines 1 to 6 were not adequate to describe their percepts. Instructions asked subjects to indicate which tunes they heard, giving them the opportunity to select as many as they wished. These changes to Butler's procedure brought our method closer to the open-ended reports used by Deutsch.

It also seemed important to ensure that subjects could adequately recognize the response alternatives when they were presented alone, if a comparison was to be made between musically trained and untrained listeners. Dowling (1973), for example, had found that subjects had difficulty recognizing interleaved melodies with overlapping pitch ranges unless they knew what melodies to listen for. We decided to give subjects binaural practice in recognizing the six notated alternatives. If they could not accurately recognize these alternatives, then little sense could be made of subjects' forced-choice responses in the critical dichotic conditions.

Because the same procedure was used in the various parts of Experiment 1 and in Experiments 2 and 3, this general procedure is reported first.

\section{GENERAL PROCEDURE}

Subjects

Subjects were from two sources: a total of 318 right-handed Macquarie University undergraduates, who participated for course credit, and 20 right-handed senior students (aged 17 to 18 years) from the New South Wales Conservatorium of Music High School. Approximately $50 \%$ of the Macquarie subjects reported some musical training in their childhood, ranging from 2 weeks to 9 years (the latter for only two subjects). The median length of training was 6 months, and all subjects indicated that now they could neither read music nor play an instrument. Initial comparisons of the results for Macquarie subjects who had 
had some musical training with those with no training indicated no differences, so their data were pooled to form a single nonmusician group. The Conservatorium students were all highly trained and competent musicians, currently involved in both learning and performing, and they formed a musician group.

Only one observation was taken from each nonmusician subject, except in Experiment 3. Because of the limited number of musician subjects, several observations were taken from each of them in Experiment 2.

Deutsch found handedness to be an important factor, so, for consistency, we did not include left-handed subjects in any analyses. In fact, we encountered only 10 left-handed subjects, and their data seemed no different from those of the righthanders.

\section{Stimuli}

Our stimuli were programmed on and recorded from a Fairlight Computer Musical Instrument. Unless otherwise indicated, the sounds were of a synthesized piano. Although Deutsch used pure sine waves, Butler was able to replicate the illusion with ordinary piano sounds, so this change in sound type should not greatly affect our ability to reproduce the illusory effect. There was also a minor difference in the timing of out sounds, compared with those of Deutsch. In the present study, the interval between the onset of successive notes was $250 \mathrm{msec}$, with the duration of each note being approximately $240 \mathrm{msec}$. Deutsch had no gaps between her tones. Independent checks confirmed correct primary frequency and simultaneity of the onset in the two channels.

\section{Procedure}

Ten sets of stereo headphones were attached to a stereo cassette recorder, with separate amplifiers and level monitors for each channel. This arrangement avoided "cross-talk" between channels and allowed group testing. Groups of between 5 and 10 subjects were presented with 20 continuous repetitions of the stimulus sequence at a comfortable listening level. They were able to hear the sequence again if desired. (In fact, subjects rarely requested a repetition.) These presentation procedures varied little from those of previous research. Although Deutsch recorded only 10 repetitions of the sequence, she presented this recording three times. Butler recorded 8 repetitions and allowed his subjects to listen to the sequence as many times as they desired. Deutsch suggested that the basic illusory percept changed with repeated presentation.

The subjects were required to choose among the six response alternatives, lines 1 to 6 in Figure 2 , or, if these inadequately described their percepts, to notate or draw the contour of additional tunes. The subjects were also asked to indicate when possible whether each perceived tune was presented in the left or right ear.
Prior to this, the subjects were given practice recognizing each of the six response alternatives presented alone and binaurally. Only when all subjects in an experimental session could correctly identify these six alternatives from binaural presentations was the experimental dichotic sequence presented. In general, this required only two presentations of the recorded 20 repetitions of each alternative.

The above procedures constituted a departure from previous experimental investigations of Deutsch's scale illusion. However, if the illusion is robust, it will persist despite these variations.

\section{EXPERIMENT 1}

Our various attempts to reproduce the scale illusion are reported as a series of parts of Experiment 1. The parts are not simply considered as treatments in the one experiment because they represent an interesting historical account of our attempts to obtain the illusion reliably.

\section{Experiment 1a}

\section{Method}

Eighteen nonmusician subjects were run through the basic procedure outlined above. Thus, they had six response alternatives, were able to indicate further possibilities if necessary, and received practice at identifying the response alternatives. The experimental stimulus was a dichotic presentation of line 5 (left ear) and line 6 (right ear).

\section{Results}

The first line of Table 1 shows the percentage distribution of subjects' responses. The figures in Table 1 represent the percentage of subjects who indicated a particular pair of tunes. $\mathrm{A}, \mathrm{B}$, and $\mathrm{C}$ refer to the paired alternatives shown in Figure 2. $(A+B),(A+C)$, and $(B+C)$ indicate alternative groupings; for instance, $(B+C)$ refers to pairing one line of $B$ with one line of $C$ (e.g., line 4 plus line 5 ).

The data show only 28\% reporting Deutsch's full illusion, B, and a great deal of variation. The "other" category responses were from four subjects, who reported a partial illusion, three combining one line of $B$ with an individually created tune and one reporting only one tune (line 4). In fact, $50 \%$ of our subjects, compared with the $17 \%$ of right-handers reported by Deutsch, heard

Table 1

Percentages of Subjects Selecting Various Response Alternatives in Experiment 1

\begin{tabular}{|c|c|c|c|c|c|c|c|c|c|c|c|}
\hline \multirow[b]{2}{*}{ Experiment } & \multirow[b]{2}{*}{ Group } & \multirow[b]{2}{*}{$\mathrm{n}$} & \multirow{2}{*}{$\begin{array}{l}\text { Response } \\
\text { Alternative }\end{array}$} & \multirow[b]{2}{*}{ Practice } & \multicolumn{7}{|c|}{ Percept Chosen } \\
\hline & & & & & $\mathbf{A}$ & B & $\mathrm{C}$ & $A+B$ & $A+C$ & $\mathrm{~B}+\mathrm{C}$ & Other \\
\hline $1 a$ & & 18 & $6+$ & $\mathbf{Y}$ & 6 & 28 & 6 & & 11 & 28 & 22 \\
\hline $1 b$ & & 22 & $6+$ & $\mathbf{N}$ & 5 & 14 & 14 & 14 & 9 & 27 & 18 \\
\hline $1 c$ & 1 & 18 & 6 & $Y$ & 6 & 25 & 17 & & 6 & 44 & \\
\hline $1 \mathrm{c}$ & 2 & 16 & 6 & $\mathbf{N}$ & & 31 & & & 25 & 44 & \\
\hline $1 \mathrm{c}$ & 3 & 15 & 6 & $\mathrm{Y}$ & 7 & 7 & 20 & 13 & 13 & 40 & \\
\hline $1 \mathrm{~d}$ & 1 & 13 & 3 & $\mathrm{Y}$ & 15 & 54 & 31 & & & & \\
\hline $1 \mathrm{~d}$ & 2 & 25 & 3 & $\mathbf{N}$ & 12 & 80 & 8 & & & & \\
\hline le & & 20 & 3 & $\mathbf{N}$ & & 100 & & & & & \\
\hline
\end{tabular}

Note- $Y=$ yes; $N=$ no. See text for details. 
only a partial illusion [indicated by $(A+B)$ plus $(B+C)$ plus "other" responses]. Perhaps the changed procedure, from shadow-singing to recognition, gave more scope for those subjects who could hear only one tune clearly, and so had a partial illusion, to indicate both this clear percept and their recognition that some other sounds were also present. Indeed, some of these subjects commented that they chose the second of their melodies on the basis of only a few notes.

Thus, although $78 \%$ of subjects reported hearing at least half of the illusion, only $28 \%$ of subjects perceived the full illusion Deutsch reported. Of those nine subjects who heard only half of the illusory percept, B, five heard the higher pitched stream and four heard the lower pitched stream. While our results are not as clear-cut as those of Deutsch, even hearing half of the illusion (i.e., either line of B) does involve streaming by pitch.

Unlike Deutsch, we did not find that subjects hearing the full, or partial, illusion tended to localize the higher sounds in the right ear. Only two of the five subjects reporting the full illusion located line 3 in the right ear. The four subjects whose partial illusion included the lower pitched line 4, all placed that tune in the left ear. Although we did not reverse headphones as a control here, Deutsch's results implied that the localization effect would occur regardless of headphone position.

\section{Experiment 1b}

\section{Method}

Because comments from some nonmusicians in Experiment la had suggested that providing practice with the response alternatives may have confused rather than helped them, 22 nonmusician subjects were tested without this practice. In other respects, the procedure was unchanged from Experiment 1a. The subjects were given a verbal description of items 1 to 6 and were asked to imagine the sounds that the notation represented. These instructions dealt with the contours of the six melodies and explained the musical notation. The subjects generally reported that this was useful.

\section{Results}

As the second line of Table 1 suggests, the results did not differ markedly from those of Experiment $1 \mathrm{a}\left[\chi_{6}^{2}=\right.$ $4.23, p>.05$; although some expected frequencies for this and later chi-square tests are quite small, Fienberg (1980) reports Monte Carlo studies which support their use]. Only $14 \%$ of subjects in Experiment $1 \mathrm{~b}$ showed the full Deutsch scale illusion by responding B, and these three subjects all localized line 3 in their right ear. The "other" category consists of four people who drew their own versions of a melody that went with one line of B. Again, a majority of listeners (59\%) reported half of the illusion, eight of them including the higher pitched stream in their selection of alternatives and five of them, the lower pitched stream. There were no clear trends in terms of the localization of either of these streams in either ear.

\section{Experiment 1c}

\section{Method}

Our results so far have been much more variable than Deutsch's, with few of our subjects reporting her full illusion, lines 3 and 4 . In Experiment 1c, given that so few subjects had at tempted to draw their own versions of the sequence, and in an attempt to reduce some of this variability, subjects' response alternatives were restricted to items 1 to 6 . This experiment involved three groups of nonmusicians: 18 in Group 1 were run with binaural practice with the response alternatives, 19 in Group 2 were run without practice, and 16 in Group 3 were given practice but were instructed to try to listen to the tunes together in the center of their heads, rather than concentrating separately on each ear. This last manipulation was to check whether our instructions to concentrate on each ear separately had contributed to our failure to reproduce the illusion, even though our initial instructions had been modeled on Deutsch's.

\section{Results}

Again, as Table 1 shows, relatively few subjects (between $7 \%$ and $31 \%$ ) reported the full illusion, B. The two groups with practice exhibited greater variability than the group without practice, although this effect was not reliable with our sample sizes $\left(\chi_{5}^{2}=7.58, \mathrm{p}>.05\right)$. It seems that the more people know about the response alternatives, the more variable their percepts of the test stimulus. In addition, asking subjects to listen to the tunes together in the center of their heads (Group 3) tended to reduce the percentage of subjects hearing the full scale illusion. In each group, however, between $44 \%$ and $53 \%$ of subjects reported a partial illusion. Again, about half reported hearing the higher pitched stream of $B$, and half, the lower pitched stream, with no pattern of the higher or lower stream being heard consistently in the right or left ear. Thus, even though be. tween $60 \%$ and $75 \%$ of subjects reported hearing one or both of Deutsch's "illusory" streams, we were still unable to reproduce her much stronger findings of $70 \%$ reporting both streams simultaneously.

\section{Experiment 1d}

\section{Method}

In a further attempt to get closer to the results of Deutsch and of Butler, we restricted subjects to just three response alternatives, A, B, or C. This is the procedure that Butler had used. Group 1 had 13 nonmusicians who were given practice and Group 2 had 25 nonmusicians who were not given practice.

\section{Results}

As shown in Table 1, $80 \%$ of the no-practice group selected B, showing a strong scale illusion and replicating Deutsch's results with our slightly different stimulus and procedures. Once more, practice increased variability, but again the effect of practice was not significant $\left(\chi_{2}^{2}=3.71, p>.05\right)$. Although the majority of subjects in the practice condition chose $B$, a substantial minority, $31 \%$ chose $\mathrm{C}$, suggesting streaming by ear. All Group 1 subjects hearing the illusion localized the higher 
pitched tune in the left ear, a result contrary to Deutsch. Of the 20 subjects in Group 2 reporting B, half heard the higher pitched line 3 in the left ear and half in the right ear.

\section{Experiment le}

Because we later wanted to use musician subjects in other conditions, it seemed important to replicate this last finding with them. Hence, Experiment 1d was replicated using 20 musicians. Practice recognition of the response alternatives was omitted, since all the musicians could interpret the musical notation.

As Table 1 indicates, the results were extremely simple: all subjects reported hearing $B$, the scale illusion. Although they had been encouraged to make any additional comments about their percepts, none of the musician subjects suggested that B did not exactly fit their percept. It is interesting that, under similar circumstances, only 52\% of Butler's musicians selected B, although Butler's subjects had previously musically notated their percepts in a more open-ended test.

In contrast to the nonmusicians, but consistent with Deutsch's findings, $90 \%$ of the musicians localized the higher tune in the right ear and the lower tune in the left ear $(p<.001$, using binomial test and taking $50 \%$ as indicating no left-right bias).

\section{Discussion}

Despite differences between Deutsch's and our stimulus parameters and procedures, we have been able to "replicate" the Deutsch scale illusion, but only when subjects' response alternatives were sufficiently restricted and when familiarity with these alternatives was limited (Experiments 1d and 1e). When our subjects were given the opportunity to respond in a more openended way (Experiments 1a and 1b), a situation closer to Deutsch's free report method, our results varied considerably from Deutsch's.

The difficulty in reproducing Deutsch's results sug. gests that the scale illusion is not a robust phenomenon. When presented with simultaneous dichotic presentations of lines 5 and 6 , most subjects seem to hear either line 3 or line 4 , but not necessarily both these tunes as Deutsch reported. This conclusion is supported by the fact that the $80 \%$ of subjects reporting the illusion in Experiment 1d matches closely the 78\%,73\%, and 60\% to $75 \%$ of subjects reporting either a whole or partial illusion in Experiments $1 \mathrm{a}, 1 \mathrm{~b}$, and 1c, respectively. Perhaps the "illusion" appears in Experiment 1d because those subjects who hear one part of B decide that $B$ is the best of three, not particularly accurate, alternatives available. Another factor which may bias the results in the "illusory" direction is that some subjects apparently did not regard alternative $\mathrm{C}$, the stimulus actually presented, as a realistic alternative. It is, after all, an unusual sequence for western music, as several of the musicians commented. This also means that $\mathrm{C}$ would be especially difficult to sing, yet Deutsch asked her subjects to indicate their percepts by singing.

Although part of our difficulty in reproducing Deutsch's results could be that we used complex musical stimuli rather than pure tones, this does not seem to fully account for our difficulty. Butler (1979) has previously used piano notes as stimuli for the scale illusion and reported results similar to Deutsch's. In any case, neither Deutsch nor Bregman have restricted their explanations of streaming to pure tones. Thus, the use of complex tones should not have prevented finding the scale-illusion effect if the initial theories of the effect were correct.

The nonmusicians' localization results in Experiment 1 did not match Deutsch's finding that righthanded subjects tended to hear the higher pitched stream, line 3, in the right ear and line 4 in the left ear. Over all parts of Experiment 1, only 57\% ( $>.23$, using binomial test) of those nonmusicians who reported both lines 3 and 4 reported line 3 in the right ear and line 4 in the left. Of nonmusicians who reported line 3 without line 4 , or vice versa, only $47 \%$ reported line 3 in the right or line 4 in the left ear. The reasons for this difference between our results and those of Deutsch become clearer when the musicians are considered, $90 \%$ of whom reported line 3 in the right ear and line 4 in the left. Perhaps Deutsch's subjects were more skilled musically, like our musicians. Why musicians exhibit such localization effects is unclear, although it may be related to the greater degree of cerebral hemispheric specialization for musical stimuli attributed to musicians (Gates \& Bradshaw, 1977).

It is puzzling to note the effects of practice. We reproduced the illusory effect strongly only when subjects were somewhat ignorant of the response alternatives. Providing practice in binaurally recognizing these alternatives tended to reduce the number of illusory percepts. Although such brief experience with different organizations of the scale-illusion stimulus could theoretically influence a parsing mechanism under Deutsch's or Bregman's theories, how this would work is unclear. Certainly, a simple biasing effect would not account for our results, and is unlikely since all response alternatives were heard equally often during practice.

These considerations raise doubts about whether the phenomenon reported by Deutsch can truly be called an illusion. Gillam (1980, p. 86) has suggested that "illusions are not conceptual but perceptual; knowing that a particular effect is illusory does not diminish the strength of the illusion." Given this description, it may be better to characterize the Deutsch scale-illusion stimulus as an ambiguous figure, analogous to a Necker cube. One explanation for the odd effects of practice in the present study is that, having been alerted to other possible organizations of the sounds, subjects found their percep- 
tions changing over time, increasing the difficulty that many had in making a decision. This is consistent with some subjects' later introspective reports. The description of this effect as an ambiguous auditory figure also seems consistent with Bregman's theory of an ongoing competition between possible organizations.

Even if the phenomenon which Deutsch reported is not very robust and is better described as the consequence of an ambiguous figure, it is evident that the results from Experiment 1 support Deutsch's arguments about the importance of pitch as a streaming dimension. As we noted earlier, the perception of either half of the effect, that is, of either line 3 or line 4, involves auditory streaming based on pitch, and between $60 \%$ and $78 \%$ of our subjects reported at least one of these lines. Moreover, our finding that $80 \%$ to $100 \%$ of subjects in Experiments $1 \mathrm{~d}$ and $1 \mathrm{e}$ responded by selecting alternative B is a large effect. It was this effect that we tried to systematically alter in our subsequent investigations of auditory streaming. If the effect is solely artifactual and due to limiting response alternatives and/or inducing demand characteristics, then the systematic manipulations of percepts attempted in Experiment 2 will not be possible.

\section{EXPERIMENT 2}

Following Bregman's proposal that streaming involves competition among alternative organizations, Experiment 2 was designed to explore changes in subjects' perceptions of the scale-illusion stimuli when some of the possible variations of tune, loudness, and timbre were introduced. A previous study (Butler, 1979) had found that even when sounds to one ear were louder than they were to the other, the illusion still persisted for musicians, supporting Deutsch's contention that pitch is the dominant dimension in this sequence. However, a more systematic manipulation of stimulus parameters may produce some instances of pitch being over- ridden by other streaming dimensions. If, for example, two different instruments played $\mathrm{C}$, one playing line 5 in the right ear and the other line 6 in the left ear, would subjects group the sounds by instrument/timbre and ear and so report $\mathrm{C}$ rather than streaming by pitch and hearing $\mathrm{B}$ ?

The leftmost section of Table 2 lists the eight variations (a to $h$ ) introduced to the basic scale-illusion sequence, and sets out for each variation the competing perceptual organizations $(A, B$, or $C$ ) implied by possible streaming dimensions considered individually. If pitch is the only, or dominant, streaming dimension used, then subjects should report $B$ regardless of the changes in the stimuli. If tune is important, then A should be chosen, since it is the best known of the three "tunes," being a fundamental western musical scale. If ear of presentation, loudness, or timbre are important dimensions, subjects should choose $\mathrm{A}$ or $\mathrm{C}$, depending on the sequence. (Predictions and results for the standard scale-illusion stimulus are included in Table 2 for comparison.)

The musical sequences were so arranged that streaming by different dimensions would result in different percepts. In all of the sequences, pitch as a cue to streaming was weighed against at least two dimensions, implying a percept other than B. Bregman's account would lead one to expect that streaming by pitch would not always occur. However, rather than generally invoking ad hoc gestalt principles to explain the final outcome of the parsing processes, some more explicit alternative explanation could be correct. It might, for example, be the case that when streaming by one dimension would lead to one percept and streaming by two others would lead to a different percept, then the percept "predicted" by the two dimensions would result. That is, the auditory parser might simply choose the percept which has the most streaming cues suggesting it. This uses a model similar to the logogen model in word perception (e.g., Morton, 1979) or the Pandemonium pattern-recognition model (Lindsay \& Norman, 1972).

Table 2

Stimuli, "Predictions," and Results in Percentages for Experiments 2 and 3

\begin{tabular}{|c|c|c|c|c|c|c|c|c|c|c|c|c|c|c|c|}
\hline \multirow[b]{3}{*}{ Stimuli (See Text for Full Account) } & \multirow{2}{*}{\multicolumn{5}{|c|}{$\begin{array}{c}\text { Response Alternative } \\
\text { Predicted Entirely } \\
\text { on the Basis of* }\end{array}$}} & \multicolumn{10}{|c|}{ Percentage of Each Response Alternative } \\
\hline & & & & & & \multicolumn{4}{|c|}{ NM } & \multicolumn{3}{|c|}{$\begin{array}{c}M \\
(n=20)\end{array}$} & \multicolumn{3}{|c|}{$\begin{array}{c}\text { NM3 } \\
(n=37)\end{array}$} \\
\hline & 1 & 2 & 3 & 4 & 5 & $\mathbf{n}$ & A & B & $\mathrm{C}$ & $\mathbf{A}$ & B & $\mathrm{C}$ & A & B & $\mathrm{C}$ \\
\hline Basic Scale Illusion: 5 -left, 6 -right** & B & $\mathrm{C}$ & & & $\mathbf{A}$ & $25+$ & 12 & 80 & 8 & & 100 & & 22 & 78 & \\
\hline (a) 1 teft, 2 -right & B & & & & A & 15 & 93 & 7 & & & & & 19 & 70 & 11 \\
\hline (b) 5 teft \& loud, 6 tight \& soft & B & $\mathrm{C}$ & C & & $\mathbf{A}$ & 17 & & 76 & 24 & & & & & & \\
\hline (c) 5-right, 6 teft, alternately loud \& soft in each ear & $\mathbf{B}$ & $\mathrm{C}$ & A & & $\mathbf{A}$ & 24 & 54 & 21 & 25 & 15 & 70 & 15 & 59 & 30 & 11 \\
\hline (d) 1 left \& loud, 2-right \& soft & B & A & $\mathbf{A}$ & & $\mathbf{A}$ & 9 & 100 & & & & & & & & \\
\hline (e) 5-left piano, 6-right saxophone & B & $\mathrm{C}$ & & $\mathrm{C}$ & $\mathbf{A}$ & 16 & 13 & 56 & 31 & & 55 & 45 & 5 & 57 & 38 \\
\hline (f) 5-left ocarina, 6-right saxophone & B & $\mathrm{C}$ & & $\mathrm{C}$ & A & 23 & & 49 & 51 & & & & 3 & 62 & 35 \\
\hline (g) 5 teft 1 st piano, 6 -right 2 nd piano & B & $\mathrm{C}$ & & C & A & 15 & & 7 & 93 & & 50 & 50 & & 32 & 68 \\
\hline (h) 5teft, 6-right, alternately piano \& saxophone in each ear & B & $\mathrm{C}$ & & $\mathbf{A}$ & $\mathbf{A}$ & 15 & 87 & 7 & 6 & 30 & 35 & 35 & 76 & 5 & 19 \\
\hline
\end{tabular}

Note $-N M=$ nonmusicians; $M=$ musicians; $N M 3=$ nonmusicians from Experiment $3 . \quad * 1=$ pitch; $2=$ ear; $3=$ loudness; $4=$ timbre; $S=$ tune. $\quad * *$ i.e., line 5 from Figure 2 presented to left ear and line 6 presented to right ear. tThese are the results from Group 2 , Experiment $1 d$, as reported in Table 1. 


\section{Method}

Following the findings in Experiments 1d and le, subjects in Experiment 2 were given no practice with response alternatives and were asked to select $\mathrm{A}, \mathrm{B}$, or $\mathrm{C}$, whichever was the closest representation of the sounds they heard, and then to provide written comments to qualify their choices, if necessary. We found, in pilot testing, that it often took some time before a clear percept developed. Subjects were warned of this and encouraged to wait until the end of the 20 repetitions before making their final choice.

Each of the new nonmusician subjects contributed only one observation, while the 20 musicians, who had participated in Experiment 1e, each contributed several observations. It was not possible to test the musicians with all of the stimuli.

All musical sequences included dichotic repetitions of the $C$ major scale but in various forms described below (the lowercase characters refer to corresponding tows of Table 2, and, as before, the line numbers refer to Figure 2).

\section{Variation on "Tune" Dimension}

(a) Here the descending scale (line 1) was played to the left ear, and the ascending scale (line 2) to the right ear. If subjects were to stream entirely by pitch, they would hear B. However, streaming by ear or tune would lead to $A$.

\section{Variations on Loudness Dimension}

(b) Line 5 was presented loudly to the left ear and line 6 softly to the right ear, thus adding an extra (loudness) cue "predicting" a $\mathrm{C}$ response.

(c) Lines 5 and 6 were presented to the left and right ears, respectively, but within each ear the notes alternated, loud then soft, such that when a loud note was presented in the right ear, a soft note was presented in the left ear (see Figure 3). If intensity differences were important, so that subjects grouped the loud and soft notes separately, they should report hearing A, because the loud notes form the descending scale and the soft notes form the ascending scale. Thus, tune and loudness are both cues to an $A$ response in this sequence.

(d) This variation was similar to (a) but with line 1 presented loudly to the left ear and line 2 presented softly to the right ear. This provides three cues to an A response.

\section{Variations on Timbre Dimension}

(e) Line 5 was played by a piano in the left ear, and line 6 was played by a saxophone in the right ear. (As mentioned earlier, all instrumental variations were generated on a Fairlight CMI synthesizer.) Cues to a $\mathrm{C}$ organization arise from differences in timbre and ear of presentation. If subjects were to stream by pitch on this sequence, they would be combining notes played by different instruments to form one high- and one low-pitch melody.

(f) This variation was similar to (e) except that line 5 presented to the left ear was played by an ocarina and line 6 presented to the right ear was played by a saxophone.

(g) This variation was similar to (e) except that line 5 presented



Figure 3. Arrangement of lines 5 and 6 in stimulus $c$ so that alternate notes were presented loud and soft. Note how the combination of pitch and loudness can produce the four streams shown as upper- and lowercase $L s$ and Ss. ( $L$ or $I=$ presented loudly; $S$ or $s=$ presented softly.) to the left ear was played by a traditional piano and line 6 presented to the right ear was played by a harpsichord-like piano.

(h) In the final sequence, the instruments were again varied, combining piano and saxophone playing $C$. But this time, rather than having all line 5 played by one instrument and line 6 by the other, alternate notes on each line were played by piano and then saxophone [analogously to the loudness manipulation in (c) above and depicted in Figure 31. As a saxophone note was presented to the right ear, a piano note sounded simultaneously in the left ear. To group the sounds of each instrument would require switching from ear to ear on successive notes. If timbre is used as the cue to organization, then the subject should respond $\mathrm{A}$, since the sounds played by the piano and saxophone add up to the descending and ascending scales, respectively. Streaming by tune adds more weight to an A response. Alternatively, the sounds could still be organized by pitch, response B, although this would mean combining piano and saxophone sounds. The other possibility is streaming by ear, which would give $C$.

\section{Results}

As in Experiment 1, data from nonmusicians who had had limited musical training were pooled with data from those with no training because a preliminary examina. tion of the data had revealed no systematic differences between them.

\section{Nonmusicians}

The first panel of results in Table 2 contains the results of the nonmusicians. It reveals that, although the pitch dimension still appeared to be important for stimuli $b$ and $e$, auditory streaming was not always based on the dimension of pitch. Stimulus parameters significantly affected response distributions $\left(\chi_{14}^{2}=\right.$ $117.7, p<.001)$. A comparison of the nonmusicians' results with the "Predictions" panel of Table 2 indicates that in most cases the modal response alternative chosen was the one "predicted" by the majority of potential streaming dimensions. For example, for stimulus d, streaming by pitch alone would lead to response alternative $B$, while streaming by either ear, loudness, or tune would lead to response alternative $A$. In fact, for stimulus d, 100\% of nonmusicians chose response alternative A. Similarly, for stimulus g, $93 \%$ of subjects gave response $\mathrm{C}$.

However, this simple account is not fully supported by the results because, with stimuli $b$ and $e$, the modal response is not that predicted by the majority of potential streaming dimensions. For stimulus $b$, streaming by pitch persisted despite a difference in the intensity of sounds to each ear. This was consistent with Butler's (1979) earlier finding. In addition for stimulus $f$, two dimensions, ear and loudness, predict a $\mathrm{C}$ response, but only $51 \%$ of the subjects gave that response and $49 \%$ still responded B. Despite the complex pattern of these results, one point is extremely clear. It is possible to vary the stimulus attributes of Deutsch's basic stimulus in order to produce streaming based on several stimulus dimensions rather than just pitch. This supports an account of 
auditory streaming which sees streaming as resulting from a competition between several possible perceptual organizations.

\section{Musicians}

The performance of the musicians (see the second results panel of Table 2) differed from that of the nonmusicians in a number of ways. First, as mentioned previously, the musicians were tested on only four (c, e, $\mathrm{g}$, and $\mathrm{h}$ ) of the nine stimuli, and the same subjects listened to each of these stimuli. For these four stimuli, the musicians produced response patterns quite different from their responses to the standard scale-illusion stimulus. This provides further evidence for a "competition among alternatives" account of streaming.

There were considerable differences between the results of the musicians and the nonmusicians for each of the four stimuli. These differences were significant for stimuli $\mathrm{c}, \mathrm{g}$, and $\mathrm{h}(\mathrm{p}<.01$ for all relevant chi-squares) but not for stimulus e $\left(\chi_{2}^{2}=2.93, p>.05\right)$. The musicians' modal response did not follow the prediction of the majority of stimulus dimensions, in contrast to that of the nonmusicians. Indeed, the musicians' modal response was always $B$. This bias toward $B$ may reflect their expectations of a standard tune. The case of one musician subject is interesting here. She felt that her original response, B, was not entirely accurate, and so she tried to notate her percept carefully, note by note, over several stimulus repetitions. When finished, she suddenly realized that she had notated item $C$. This indicates that at least some of the musicians did not expect to hear such a thing as $\mathrm{C}$. Some of the bias shown by musicians to response $\mathrm{B}$ may also have been due to the fact that they judged several stimuli in one session and that the first stimulus was the standard scale illusion sequence used in Experiment 1e.

\section{Localization of Streams}

Of the nonmusicians who heard B for any one of the Experiment 2 stimuli, 78\% localized line 3 in the left ear, which is significantly in the direction opposite to Deutsch's results $(p<.001$ for binomial test). For the musicians, $57 \%$ of the B responses were associated with hearing line 3 in the right ear. Again, the musicians showed a slight effect in the direction of Deutsch's results. However, Experiments 1 and 2 together show no clear localization effects.

\section{EXPERIMENT 3}

Bregman's theory suggests that similar parsing heuristics would operate to determine auditory streaming irrespective of whether presentation is dichotic or binaural, except of course that ear of input is less relevant for binaural conditions. This predicts much the same pattern of results with binaural as with dichotic presentations of our stimuli. Experiment 3 was designed to test this prediction.

\section{Method}

In a repeated-measures design, 37 new nonmusicians were tested in gtoups of five to eight. Each group heard binaural presentations of the stimulus used in Experiment 1 and of six. stimuli from Experiment 2 in a different order. In other respects, the procedure was the same as that in Experiment 2. Subjects had to respond either $\mathrm{A}, \mathrm{B}$, or $\mathrm{C}$, but they were not asked to indicate the ear in which they heard each stream.

Strictly speaking, this was a silly task, as the sounds presented in the binaural sequences were the same except when timbre differences were introduced. That is, an acoustic rendition of any of the three response alternatives A, B, and C, would be physically the same. Our subjects, however, had no doubt about their responses and produced the remarkably consistent results shown in Table 2.

\section{Results}

The pattern of results for each stimulus were not significantly different from those obtained in Experiment 2 (for all relevant chi-square, $p>.05$ ), except for stimulus a $\left(\chi_{2}^{2}=24.6, p<.001\right)$. For all stimuli except stimuli $a$ and $f$, the binaural presentations used in Experiment 3 led to the same modal response as the dichotic presentations of Experiments 1 and 2. The slight and nonsignificant deviation with respect to stimulus $f$ cannot be afforded much importance, since the modal and second modal responses in Experiment 2 had such close response rates (51\% and 49\%). The difference between the two experiments for stimulus a is also understandable because, with binaural presentations, the ear of input cue to streaming is not operative. Hence, the ear and loudness "predictions" of A which led to a 93\% A response to the dichotic presentations of Experiment 2 are not both operating in Experiment 3. This probably accounts for the reduced $\mathbf{A}$ response in Experiment 3.

For the standard stimulus used in Experiment 1, the nonmusicians from Experiment 3 performed comparably to those from Experiment 1, as Table 2 indicates. However, in Experiment 3 no subjects reported hearing alternative $\mathrm{C}$, while a small percentage did in Experiment 1.

Although the results of Experiment 3 do not exactly match those of Experiment 2, this may be because each subject listened to all the stimuli in Experiment 3 . The results of Experiment 3 do show that, even for binaural presentations, it is possible to induce streaming by a variety of stimulus dimensions. With both dichotic and binaural presentations, streaming could be the result of a process which decides among alternative potential organizations.

\section{GENERAL DISCUSSION}

Experiment 1 established that it was both possible and difficult to reproduce Deutsch's musical scale "illusion" with complex musical sounds rather than pure tones and with variations in experimental procedure. It did seem questionable whether the effect was an illu- 
sion or an ambiguous figure: it certainly is not a robust effect comparable with a standard visual fllusion such as the Miller-Lyer. Throughout Experiment 1, we found that between $60 \%$ and $80 \%$ of subjects reported at least half of the illusion, indicating the dominance of pitch streaming for this sequence. Experiments $1 \mathrm{~d}$ and le provided a strong effect which formed the basis for our subsequent examination of auditory streaming in Experiments 2 and 3 . In these experiments, we were able to alter subjects' percepts so that they did not always hear $B$, the percept resulting from streaming by pitch. Our manipulations of both listener characteristics and stimulus parameters support Bregman's argument that auditory streaming is the result of a competition among several, more or less likely, perceptual organizations.

The different patterns of results for musicians and nonmusicians reflected differences in musical experiences. Although the nonmusicians' modal response varied from stimulus to stimulus, the musicians mostly reported hearing $B$ for all stimuli. The precise reasons for these differences between musicians and nonmusicians are unclear. Perhaps the particular experience of the musicians leads them to expect a tune like B or perhaps for some other reason they used pitch as a basis of streaming more often than did the nonmusicians. These findings highlight the importance of considering the listener's level of musical skill in all such experiments on auditory streaming.

A slightly different view of these differences between musicians and nonmusicians arises from comments written by some of the musicians. For instance, when hearing sequence $h 14$ of the 20 musicians reported being frustrated by the forced-choice procedure. They described their percept as having four streams rather than two (these four streams correspond to the upperand lowercase lines of $\mathrm{Ls}$ and of Ss illustrated in Figure 3, except that Figure 3 depicts four lines defined by loudness and pitch rather than by timbre and pitch). Thus, the musicians' choices among A, B, and C must sometimes be regarded as arbitrary. The musicians also had trouble with stimulus c. Although $70 \%$ selected B, 8 of these 14 reported hearing the two melodies switching from ear to ear and found that confusing. In addition, two other musicians noticed more than two streams for this stimulus.

Several aspects of our results need further investigation. First, what we called a "tune" dimension was important in explaining our results. This dimension seems to be based on the experience of subjects with the $\mathrm{C}$ major scale, although physical parameters such as melodic direction and tonal closeness of successive notes may also be involved. Further research is needed on this "tune" dimension in auditory streaming. In this study, for instance, the trained musicians showed a weaker tendency to stream by "tune" (response A) than did the nonmusicians. While melodic direction and tonal closeness may be more regular in a scale, these features need not be indicative of a good "tune." Perhaps a scale is a less typical "tune" for musicians than for nonmusicians. We restricted the melodic sequences used in this study to the standard scale-illusion sequence, and so were not able to systematically test the conditions that would induce streaming by a "tune" dimension.

Second, although subjects in Experiments 2 and 3 streamed by various dimensions, pitch still appeared to be a more influential dimension than the others tested in this study. This was so for the musicians especially, and it may also explain aspects of the nonmusicians results, as suggested below.

The results of all three experiments also bear on Deutsch's report that her right-handed subjects reported the higher pitched stream, line 3 , in the right ear and the lower stream, line 4 , in the left ear regardless of actual ear of input of the stimuli, lines 5 and 6 . Our righthanded nonmusicians did not exhibit such strong localization effects. If anything, they showed the reverse effect, hearing the higher pitched line in the left ear. The musicians' results, however, did tend toward Deutsch's localization effects. This trend was especially strong ( $90 \%$ in this direction) in Experiment 1e, which most closely replicated Deutsch's results. The effect was still evident but was much weaker in Experiment 2 for $B$ responses $(58 \%)$. Perhaps, as we surmised earlier, our musicians produced results closer to Deutsch's because her subjects were also musically well trained.

A major question which our results do not fully answer concerns the exact nature of the parsing mechanism: what sort of processing goes on to determine the perceptual organization embodied in subjects' percepts. Without being explicit, both Bregman and Deutsch suggested that well-established gestalt principles may be involved in this processing. The suggestion offered below is not incompatible with this suggestion, but is the beginning of a more explicit account.

For the nonmusicians, all patterns of response except to the standard scale illusion stimulus and to stimuli b and e fit a process that involves a tendency to perceive that stimulus organization (for the parser to choose that organization) which the majority of the potential streaming dimensions considered on their own would "predict." Perceptions of the three stimuli that do not fit this view can be explained by making two additional assumptions. The first of these assumptions is that pitch is a more salient, or more heavily weighted, dimension than the others. As mentioned above, Deutsch's results and ours support this assumption, at least for such stimuli as the scale-illusion sequence. The second assumption is that there is a general bias against responding $C$ in this situation. Together, these additional assumptions explain why, for stimuli $b$ and $e$, subjects tend not to respond with the majority prediction $C$ but rather with $B$.

A problem with any arguments along this line is that, in Experiments 1,2, and 3, streaming by pitch and choosing response alternative $\mathrm{B}$ have been confounded. 
Any evidence supporting a heavier weighting for pitch as a streaming dimension could equally well be taken as evidence for a response bias toward B. Nevertheless, there is some evidence of a response bias toward B in the comments and percepts of the musicians. This evidence also shows the bias against $\mathrm{C}$, which was our second assumption above. As mentioned earlier, the musicians consistently chose B as their modal response, although their pattern of response certainly did change across stimuli, following in many respects the results of the nonmusicians. This may suggest a response bias towards B, or it may indicate that pitch is the most salient dimension. Certainly, $C$ is a most atypical tune in western music, as several of our musicians and some nonmusicians commented. This, and the fact that most tunes do not include scales like $A$ even though they are based on such scales, could lead to a bias towards $B$.

Experiments 1, 2, and 3 can do no more than provide the first steps toward detailing the processes involved in an auditory parser. There are some additional puzzling aspects of our results which complicate this problem. For instance, consider responses to stimuli e, f, and $g$. For each of these stimuli, two different instruments were used to play lines 5 and 6 , respectively. The only difference between these stimuli was the instruments involved: for (e), they were a piano and saxophone, for (f), a saxophone and an ocarina, and for (g), a harpsichord-like piano and a concert piano. The effect of the timbre dimension in "predicting" $\mathrm{C}$ appears very strong with the two pianos (for stimulus g, the $\mathrm{C}$ response was $93 \%$ ) but relatively slight when two more different instruments were used, for example, the piano and saxophone (for stimuluse, the $\mathrm{C}$ response was only $31 \%$ ). It seems odd that timbre is a stronger streaming dimension when two instruments closer in timbre are used. Future research may have to investigate aspects of timbre more carefully than we have been able to.

Another overriding consideration in determining the implications of our results for a proposed auditory parser is that our patterns of results are percentage distributions across subjects, while obviously the processes and tendencies involved in any parser are within individuals. A group's tendency to respond, say, B may reflect a B bias in each subject or it may simply mean that most subjects will always respond B. Making inferences about individual's processing from average results must always be done with some caution. We do have some anecdotal evidence that our group response distributions reflect individual responses that are based on a distributional process (like competition among alternative organizations). We, as experimenters, some of our more musically experienced subjects, and our informed colleagues have experienced fluctuating percepts for some of the stimuli even though one percept was always clearly dominant.

\section{REFERENCE NOTE}

1. Smith, J. You don't have to have rhythm but it helps: Musicians' and nonmusicians' reproductions of musical rhythms.
Paper presented at the Sixth Annual Experimental Psychology Conference, Australian National University, Canberra, May 1979.

\section{REFERENCES}

Bregman, A. S. Auditory streaming: Competition among alternative organizations. Perception \& Psychophysics, 1978, 23, 391-398. (a)

BREgman, A. S. The formation of auditory streams. In J. Requin (Ed.), Attention and performance VII. Hillsdale, N.J: Erlbaum, 1978. (b)

Bregman, A. S. Asking the "what for" question in auditory perception. In M. Kubovy \& J. R. Pomerantz (Eds.), Perceptual organization. Hillsdale, N.J: Erlbaum, 1981.

Bregman, A. S., \& Campbell, J. Primary auditory stream segregation and perception of order in rapid sequences of tones. Journal of Experimental Psychology, 1971, 89, 244-249.

Butlen, D. A further study of melodic channeling. Perception \& Psychophysics, 1979, 25, 264-268.

Coren, S., \& Girgus, J. S. Seeing is deceiving: The psychology of visual illusions. Hillsdale, N.J: Erlbaum, 1978.

Deutsch, D. An auditory illusion. Nature, 1974, 251, 307-309.

DEUTSCH, D. Two-channel listening to musical scales. Journal of the Acoustical Society of America, 1975, 57, 1156-1160. (a)

Deutsch, D. Musical illusions. Scientific American, 1975, 233(4), 92-104. (b)

Deutsch, D. The psychology of music. In E. C. Carterette \& M. F. Friedman (Eds.), Handbook of perception (Vol. 10): Perceptual ecology. New York: Academic Press, 1978.

Deutsch, D. Binaural integration of melodic patterns. Perception \& Psychophysics, 1979, 25, 399-405.

DEUTsCH, D. The octave illusion and the what-where connection. In R. S. Nickerson (Ed.), Attention and performance VIII. Hillsdale, N.J: Erlbaum, 1980.

DeUtsch, D., \& Roll, P. L. Separate "what" and "where" decision mechanisms in processing a dichotic tonal sequence. Journal of Experimental Psychology: Human Perception and Performance, 1976, 2, 23-29.

Dowling, W. J. The perception of interleaved melodies. Cognitive Psychology, 1973, 5, 322-337.

Dowling, W. J. Scale and contour: Two components of a theory of memory for melodies. Psychological Review, 1978, 85, 341-354.

Fienberg, S. E. The analysis of cross-classified categorical data (2nd ed.). Cambridge, Mass: The MIT Press, 1980.

Gates, A., \& Bradshaw, J. L. The role of the cerebral hemispheres in music. Brain and Language, 1977, 4, 403-431.

Gillam, B. Geometrical illusions. Scientific American, 1980, 242(1), 102-111.

JudD, T. Comments on Deutsch's musical scale illusion. Perception \& Psychophysics, 1979, 26, 85-92.

Lindsay, P. H., \& Norman, D. A. Human information processing: An introduction to psychology. New York: Academic Press, 1972.

Moray, N. Listening and attention. Harmondsworth, England: Penguin, 1969.

Monton, J. Word recognition. In J. Morton \& J. C. Marshall (Eds.), Psycholinguistic series: 2, Structures and processes. London: Paul Elek, 1979.

Power, R. P., Hausfeld, S., \& Gorta, A. Workshops in perception. London: Routledge \& Kegan Paul, 1981.

SLoвodA, J. A. Visual perception of musical notation: Registering pitch symbols in memory. Quarterly Journal of Experimental Psychology, 1976, 28, 1-16.

Slobona, J. A. Perception of contour in music reading. Perception, 1978, 7, 323-331.

(Manuscript received June 25, 1982;

revision accepted for publication September 1, 1982.) 